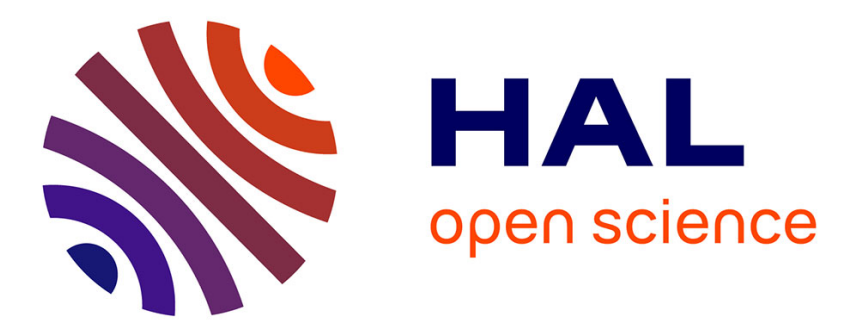

\title{
RIC-MAC: a MAC Protocol for Low-Power Cooperative Wireless Sensor Networks
}

Le-Quang-Vinh Tran, Olivier Berder, Olivier Sentieys

\section{To cite this version:}

Le-Quang-Vinh Tran, Olivier Berder, Olivier Sentieys. RIC-MAC: a MAC Protocol for Low-Power Cooperative Wireless Sensor Networks. IEEE Wireless Communications and Networking Conference (WCNC), Apr 2014, Istanbul, Turkey. pp.1944-1949, 10.1109/WCNC.2014.6952567 . hal-01097607

\section{HAL Id: hal-01097607 \\ https://inria.hal.science/hal-01097607}

Submitted on 20 Dec 2014

HAL is a multi-disciplinary open access archive for the deposit and dissemination of scientific research documents, whether they are published or not. The documents may come from teaching and research institutions in France or abroad, or from public or private research centers.
L'archive ouverte pluridisciplinaire HAL, est destinée au dépôt et à la diffusion de documents scientifiques de niveau recherche, publiés ou non, émanant des établissements d'enseignement et de recherche français ou étrangers, des laboratoires publics ou privés. 


\title{
RIC-MAC: a MAC Protocol for Low-Power Cooperative Wireless Sensor Networks
}

\author{
Le Quang Vinh TRAN, Olivier BERDER, Olivier SENTIEYS \\ IRISA/INRIA, University of Rennes 1 \\ 6 rue de Kerampont BP 80518 - 22305 Lannion Cedex, France \\ \{vinh.tran, olivier.berder, olivier.sentieys\} @irisa.fr
}

\begin{abstract}
In this study, a receiver initiated cooperative medium access control (RIC-MAC) protocol is proposed for cooperative communications to reduce the energy consumption of wireless sensor networks (WSNs). Considering a real WSN platform, the simulation results show that using the proposed RICMAC protocol in cooperative communications provides latency and energy gains as compared to multi-hop communications. However, the energy gain is shown to be reduced when the network traffic load increases. Finally, considering the impact of traffic load on energy consumption and latency, RIC-MAC is illustrated to be robust to traffic load variations in terms of latency.
\end{abstract}

\section{INTRODUCTION}

Studies on wireless sensor networks (WSNs) have impressively attracted many researchers due to advances in integrated circuit technology. WSNs are expected to be low-cost, reliable and easy to deploy. Each node in a WSN is powered by a small battery that may not be rechargeable or renewable for a long time. Therefore, the energy consumption is the most critical constraint in WSNs. To preserve energy, many efforts are proposed in the literature for energy-efficient MAC protocols, since the MAC protocol is responsible for regulating the shared wireless medium access of the networks. Among them, Low Power Listening (LPL) or preamble sampling techniques, in which regular sleep periods are scheduled for each node, are among the best strategies to satisfy the energy constraint of WSNs [1]-[5].

Cooperative relay techniques have been widely considered due to their simplicity and their good performance [6]. In cooperative relay networks, the destination receives signals from different nodes through independent fading channels. Therefore, the performance of the networks is increased, which leads to a reduction in the transmission energy [7]. This is the reason why cooperative relay is identified as a core technique to minimize total energy consumption in WSNs. Considering the parameters of RF circuits with an ideal MAC protocol, the comparison between cooperative and non-cooperative transmission schemes in terms of energy efficiency has been explored a lot in the literature [8]-[11]. In [8], the impacts of cell radius, relay position, number of relays and target data rate are investigated for cooperative systems using low-cost fixed relays. A thorough comparison on energy efficiency of single-hop, multi-hop and cooperative transmissions in WSNs is derived when the receiver is constrained by packet loss and end-to-end throughput [9]. In [10], an energy efficient cooperative multicast transmission scheme with power control is proposed to obtain a balance between system throughput and energy consumption. The application of energy-efficient cooperative techniques for intelligent transport system networks is illustrated in [11].

The above mentioned articles, however, always considered ideal MAC protocols (e.g. no packet control, no collision and no retransmission, etc.). Energy-efficiency evaluation of some MAC protocols in cooperative systems are considered in [12]-[16]. In [12], a cooperative MAC protocol combined with physical layer power/rate control is proposed to reduce energy consumption. Non-cooperative HybridAutomatic-Repeat-ReQuest (HARQ) and cooperative HARQ are considered in [13]. In [14], the HARQ protocol is analyzed in conjunction with hybrid relaying schemes from an energy efficiency perspective. A cooperative MAC (CoopMAC) for wireless LANs, in which high data rate stations assist low data rate stations in their transmission by forwarding their traffic, is proposed in [15]. CoopMAC is improved by facilitating cooperative signal combining at the destination and employing two relays in the context of a successive relaying technique in [16].

Previous works proposed MAC protocols supporting cooperative relay techniques. However, most of them extend the IEEE 802.11 standard for cooperative relay networks. Unlike other networks, WSNs are low power and lossy. Therefore, the design of low-power cooperative MAC protocols is now very essential for WSN applications. The initial work on this problem has been considered in [17]. A cooperative low-power MAC (CL-MAC) protocol for WSNs is proposed. However, CL-MAC protocol is initiated by the transmitter which may lead to bad performance under strong fading conditions [4]. Moreover, in CL-MAC, a perfect timing for node synchronization which is somehow difficult to implement in real WSN applications is strictly required. To overcome these missing gaps, in this paper a receiver initiated cooperative MAC (RIC-MAC) protocol is proposed for cooperative WSN communications.

The contributions of the paper are:

1) A new low power cooperative MAC protocol, RIC-MAC, in which the strictly required node synchronization is relaxed, is proposed to prolong the lifetime of WSNs.

2) The energy consumption is precisely evaluated through parameters extracted from a real radio platform (i.e. 
PowWow platform [18]), whereas other papers normally use an artificial energy model (e.g. [19], [20]).

3) A comparison of energy consumption between single-hop Single-Input Single-Output (SISO), multi-hop SISO and cooperative relay systems is given for ideal and real MAC protocols to show the significant impact of the MAC layer on the total energy consumption.

4) The latency is also considered, since energy consumption in WSNs is usually minimized subjected to some latency constraints. Moreover, the traffic load is also shown to have a considerable impact on energy and latency.

The rest of this paper is organized as follows. Section II presents system models. The RIC-MAC protocol, followed by energy and latency analysis, is thoroughly detailed in Section III. Energy consumption and latency simulations of the systems using ideal or real MAC protocols are derived in Section IV and, finally, conclusion is given in Section V.

\section{SySTEM MODEL}

In this paper, a typical cooperative relay model (Fig. 1) which has one source (S), one relay (R1) and one destination (D) is considered. All terminals are only equipped with one antenna. Regenerative relays are used in the multi-hop SISO and cooperative relay models. After receiving signals from the source, the relays will decode the signals and then forward the decoded signals to the destination. The relays are uniformly distributed between $\mathrm{S}$ and $\mathrm{D}$ and the distance between $\mathrm{S}$ and $\mathrm{D}$ is denoted as $d_{s d}$. We assume that all communications are performed over a flat Rayleigh fading channel and that the transmit power is equally allocated among the transmitters (e.g. source and relays). Ideal channel state information (CSI) is known at the receiver, but not at transmitters. Using a common transmission model, the signal to noise ratio (SNR) of the link from node $i$ to node $j$ is $\gamma_{i j} \propto d_{i j}^{-\alpha}$ where $d_{i j}$ is the distance between $i$ and $j$ and $\alpha=2$ is the path-loss exponent. A BPSK modulation is considered.

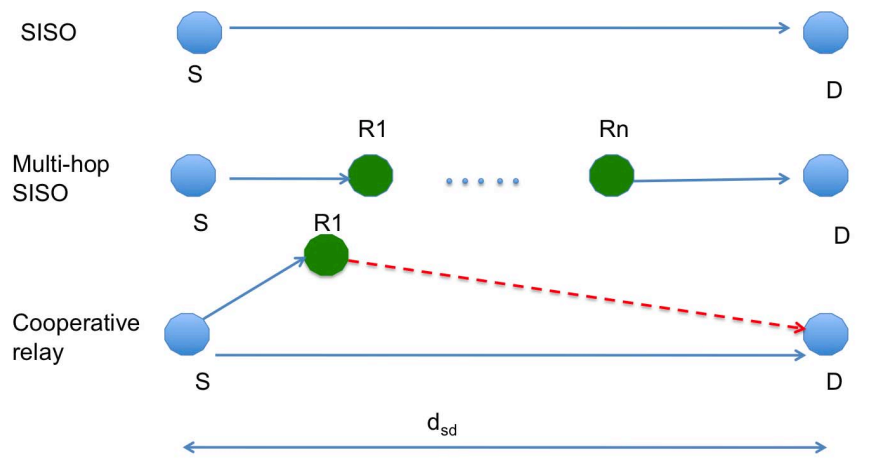

Fig. 1. SISO, multi-hop SISO and cooperative relay models

\section{RIC-MAC PROTOCOL}

\section{A. Communication protocol}

In RIC-MAC, the packet exchanges are initiated by the targeted node. Each receiver node D periodically wakes up and if the channel is free, transmits a tagged wakeup beacon (WB) and then monitors the channel for $M$ time slots to receive a request-to-send (RTS) packet. When a source node $\mathrm{S}$ needs to transmit some packets to $\mathrm{D}$, it first needs to listen to the channel and wait for the WB of $\mathrm{D}$. Then, $\mathrm{S}$ sends a buzz signal (BZ) right after the WB to initiate the communication with D. After sending BZ, S randomly chooses one of the M slots to transmit its RTS and waits for a clear-to-send (CTS) from D. If $\mathrm{D}$ does not receive $\mathrm{BZ}$, it returns to sleep to reduce energy waste of monitoring the $\mathrm{M}$ slots.

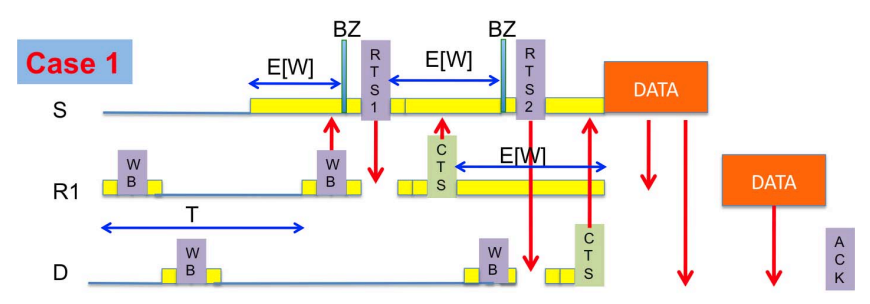

(a) relay wakes up before destination

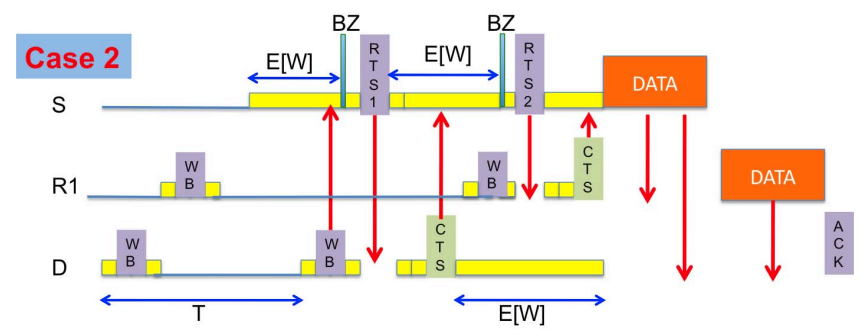

(b) destination wakes up before relay

Fig. 2. RIC-MAC protocol for cooperative communications when the relay wakes up before the destination (a) and vice versa (b)

The principle of the RIC-MAC protocol is shown in Fig. 2. Each S-D transmission takes advantage of a fixed relay node $\mathrm{R} 1$ to enhance communication performance. When $\mathrm{S}$ wakes up, it senses the channel to know whether R1 or D is awake.

- Case 1: R1 wakes up before D

1) R1 transmits WB and waits for RTS packets from $S$ if it receives BZ; otherwise it returns to sleep.

2) After receiving RTS1, R1 sends CTS to $S$ and waits for RTS/CTS exchange between S and D.

3) S sends BZ then RTS2 to D when it receives WB from D.

4) If $\mathrm{S}$ and $\mathrm{R} 1$ receive CTS from $\mathrm{D}, \mathrm{S}$ starts to transmit data to $\mathrm{D}$ and $\mathrm{R} 1$. Then $\mathrm{R} 1$ decodes data, forwards them to $\mathrm{D}$ then goes to sleep. Otherwise, $\mathrm{S}$ and $\mathrm{R} 1$ return to sleep.

5) When $D$ gets data from R1, it sends ACK to $S$ to end the communication. If $\mathrm{ACK}$ is not received by $\mathrm{S}, \mathrm{S}$ returns to sleep and waits for another period to resend data to D.

- Case 2: R1 wakes up after D.

The communication protocol is nearly the same as case 1 , but with D receiving RTS1 from $S$ and $R 1$ receiving RTS2 from S. 
In our scheme, there are three types of RTS packet (i.e. RTS0, RTS1 and RTS2).

- RTS0 is for SISO, multi-hop SISO communications.

- RTS1 and RTS2 are reserved for the cooperative relay communication.

The role of a terminal acting as a relay or a destination is included in the address field of RTS0, RTS1 and RTS2 packets. $\mathrm{X}$ may receive RTS0, RTS1 and RTS2 in the same wakeup period from different sources. To minimize the power consumption of monitoring, $\mathrm{X}$ will choose the source depending on the priority order of RTS packets (RTS2 $>$ RTS1 $>$ RTS0). If $X$ receives the same type of RTS packets (e.g. RTS0 or RTS1 or RTS2), it will randomly choose a source to make a connection by sending the CTS packet. The other sources will go to sleep and wait for another period to communicate with $\mathrm{X}$.

\section{B. Energy consumption analysis}

In our system, we assume that each node has $n$ neighbors and only one potential destination node. RTS0, RTS1 and RTS2 have the same packet length. RTS (including RTS0, RTS1 and RTS2), CTS, DATA, ACK, WB and BZ packets take respectively $T_{R T S}, T_{C T S}, T_{D A T A}, T_{A C K}, T_{W B}$ and $T_{B Z}$ seconds to transmit. The propagation delay and decoding time at R1 are assumed to be negligible compared to the transmission time. All packets except for BZ include a preamble for bit synchronization that takes $T_{a}$ seconds. $T$ is the wakeup period of nodes. The network traffic is assumed to have a Poisson distribution with mean of $\lambda$ packets per second per node.

In order to evaluate the energy consumption of the protocols, MAPLAP MAC and Gilbert-Elliot channel models [4] are considered. Let $P_{t x}, P_{r x}, P_{a q}, P_{m n}$ and $P_{s p}$ be the power consumption levels of the transceiver while transmitting, receiving, acquiring the preamble, monitoring the channel and powered off. Let $\delta_{t x}, \delta_{r x}, \delta_{a q}, \delta_{m n}$ and $\delta_{s p}$ be the average time of being in the above states for one successful communication.

The expected number of WBs transmitted in one second, $E\left[N_{W B}\right]$ is

$$
\begin{aligned}
E\left[N_{W B}\right]= & \left(1-p_{b y}\right)\left(1-\lambda^{\prime}\left(E[W]+2 M T_{R T S}\right.\right. \\
& \left.\left.+2 T_{C T S}+2 T_{D A T A}+2 T_{A C K}\right)\right) / T
\end{aligned}
$$

where $\lambda^{\prime}=\lambda /\left(\pi_{G}\left(1-p_{c o}\right)\right)$ is the scaled traffic load due to retransmissions of the entire session caused by channel fading which is represented by the average probability being in good state $\pi_{G}$, and collision rate $p_{c o} ; p_{b y}=1-(1-$ $\left.\left(E\left[N_{W B}\right] T_{W B}+\lambda\left(T_{R T S}+T_{C T S}+T_{D A T A}+T_{A C K}\right)\right)\right)^{n}$ is the probability that a node detects the channel to be busy before transmitting a WB and backs off until $T$ seconds later.

The expected waiting time until a node successfully receives a WB is

$$
E[W]=\left(\frac{1}{2 T}+\frac{p_{W B}}{1-p_{W B}} T\right) / p_{R T S}
$$

with $p_{R T S}=\left(1-p_{b y}\right)\left(1-p_{1}\right)\left(1-p_{2}\right) \pi_{G}$ the probability that an RTS is successfully responded with a CTS, where $p_{1} \approx$ $e^{-\lambda T}+\lambda e^{-\lambda T} \frac{M-1}{M} \frac{1}{2}+\frac{(\lambda T)^{2}}{2} e^{-\lambda T}\left(\frac{(M-1)(M-2)}{M^{2}} \frac{1}{3}+\frac{M-1}{M^{2}}\right)$ is the probability that RTS is collided or is not chosen by the target node and $p_{2}=1-\left(1-\lambda T_{W B}\right)^{n}$ is the probability that the expected CTS collides with a WB; $p_{W B}=1-(1-$ $\left.p_{b y}\right)\left(1-\pi_{G}\right)$ is the probability that a WB fails to be received due to busy channel or fading channel.

The total energy consumption per successful transmission $E_{\text {total }}$ for cooperative relay system with RIC-MAC can be approximated from the above expressions and can be expressed as follows:

$$
\begin{aligned}
\delta_{t x}= & 2 E\left[N_{W B}\right] T_{W B}+\lambda^{\prime}\left(\left(2 T_{R T S}+2 T_{C T S}\right.\right. \\
& \left.\left.+2 T_{B Z}\right) / p_{R T S}+2 T_{D A T A}+T_{A C K}\right) \\
\delta_{r x}= & 2 E\left[N_{B Z}\right] T_{B Z}+\lambda^{\prime}\left(\left(3 T_{W B}+3 T_{R T S}+2 T_{C T S}\right)\right. \\
& \left./ p_{R T S}+3 T_{D A T A}+T_{A C K}-\left(\frac{8}{p_{R T S}}+4\right) T_{a}\right) \\
\delta_{a q}= & \lambda^{\prime}\left(\left(8 / p_{R T S}+4\right) T_{a}\right) \\
\delta_{m n}= & 2 E\left[N_{B Z}\right] M T_{R T S}+2\left(E\left[N_{W B}\right]-E\left[N_{B Z}\right]\right) T_{B Z} \\
& +3 \lambda^{\prime} E[W] \\
\delta_{s p}= & 1-\delta_{t x}-\delta_{r x}-\delta_{a q}-\delta_{m n} \\
E_{t o t a l}= & \delta_{t x} P_{t x}+\delta_{r x} P_{r x}+\delta_{a q} P_{a q}+\delta_{m n} P_{m n}+\delta_{s p} P_{s p}
\end{aligned}
$$

where the expected number of $\mathrm{BZ}$ that a node receives in one second is $E\left[N_{B Z}\right]=\lambda^{\prime} / p_{R T S}+n \lambda^{\prime}\left(T_{C T S}+T_{B Z}\right) / T$.

In RIC-MAC, the average latency $L$ of a successful transmission (including retransmissions if necessary) is derived as

$$
\begin{aligned}
L= & 2 T_{W B}+2 M T_{R T S}+2 T_{C T S}+3 * E_{W} /\left(1-p_{f 5}\right) \\
& +p_{f 5} T /\left(1-p_{f 5}\right)+2 T_{b z},
\end{aligned}
$$

with $p_{f 5}=1-p_{R T S}\left(1-p_{b y}\right) \pi_{G}$, the handshake failure rate due to busy channel or channel fading.

\section{Simulation RESUlts}

In terms of real MAC protocols for the following simulations, whereas RIC-MAC is used for the cooperative relay system, RICER5b [5] is used for SISO and multi-hop SISO systems, since RICER5b can be considered as a reference for receiver-initiated MAC protocols. The simulations are done using a strong fading channel model with the same parameters as in [5]. BZ and DATA packets need respectively 4 and 230 bits while all other packets use 60 bits. The wakeup period $T$ is modified depending on the traffic load to get the region of interest in which the energy consumption decreases with increasing latency. Number of RTS slots is $M=2$ and neighbor nodes is $n=7$.

\section{A. Artificial energy model}

Considering ideal (Fig. 3) and real MAC protocols (Fig. 4), the comparison of energy consumption between SISO and cooperative relay systems is given based on an artificial energy 
model [19]. Fig. 3 shows that with an ideal MAC protocol, the cooperative relay system can get an energy gain of $60 \%$ in comparison with SISO system at a transmission distance $d_{s d}=30 \mathrm{~m}$. However, Fig. 4 shows that cooperative relay systems have no energy gain as compared to SISO systems due to the complex MAC protocols of cooperative systems. This demonstrates a significant impact of the MAC protocol on the total energy consumption of the systems.

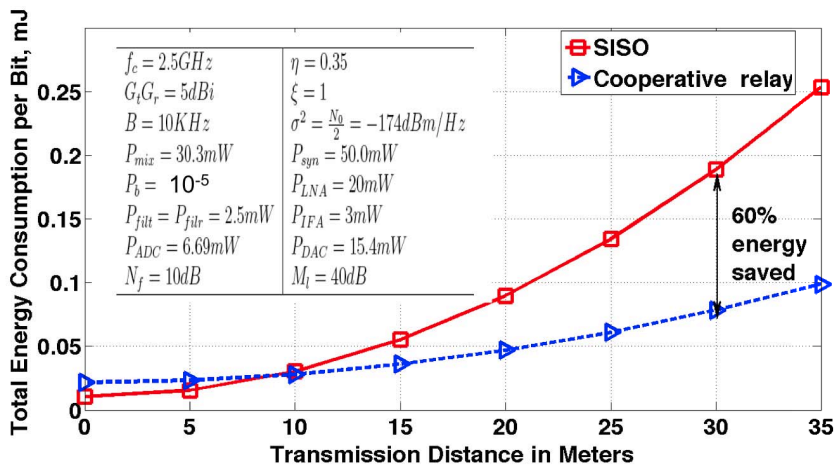

Fig. 3. Comparison of energy consumption between single-hop and cooperative relay using ideal MAC protocols (artificial energy model)

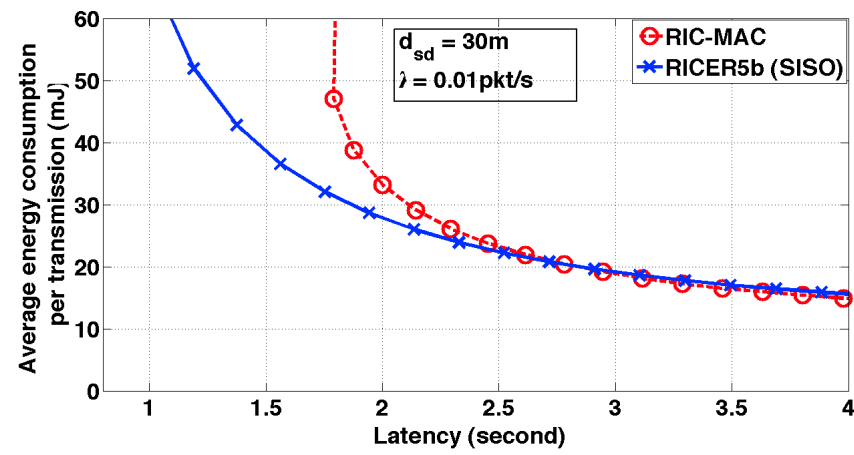

Fig. 4. Comparison of energy consumption between single-hop and cooperative relay using real MAC protocols (artificial energy model)

\section{B. Energy model based on a real platform}

The impact of the MAC protocol can be clearly demonstrated through an artificial energy model. However, this does not give us a precise estimation since it is practically impossible to perform a successful transmission at any distance. Considering a real platform, e.g. PowWow, Fig. 5 shows that each model has a maximum transmission distance depending on the desired BER, the maximum transmit power of the transceiver, i.e. CC2420 and the transmission protocol. Considering the PowWow platform, a direct SISO system with $B E R=10^{-5}$ has a maximum transmission distance of only $14.5 \mathrm{~m}$. The energy consumption comparison between SISO and cooperative relay when $d_{s d}>14.5 \mathrm{~m}$ is somehow meaningless. Since only 3-hop SISO and cooperative relay can get a transmission distance over $30 \mathrm{~m}$, they will be used to make a comparison on energy consumption. Using an ideal
MAC protocol, the cooperative relay system translates into a $35 \%$ energy consumption gain in comparison with the 3-hop SISO system (Fig. 5).

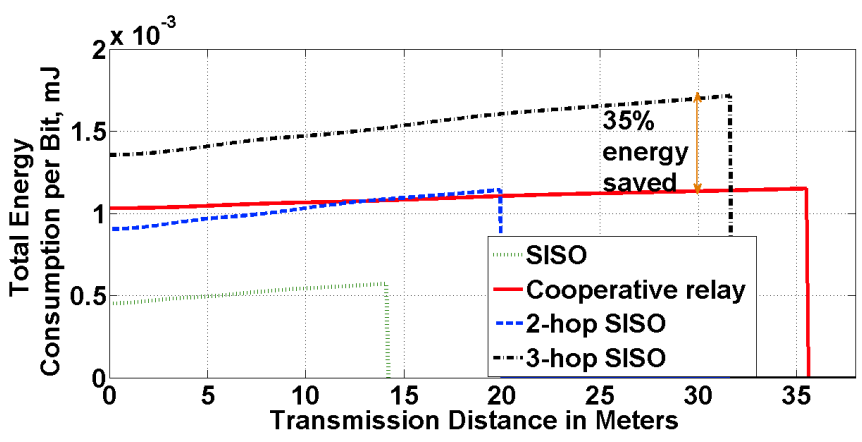

Fig. 5. Comparison of energy consumption between multi-hop and cooperative relay using an ideal MAC protocol (energy models based on PowWow platform)

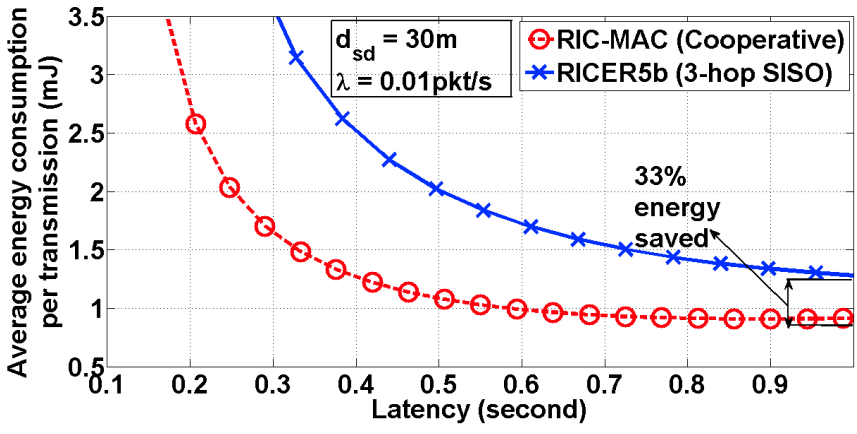

Fig. 6. Comparison of energy consumption between multi-hop and cooperative relay using real MAC protocols (energy models based on PowWow platform) in low traffic load networks

The energy consumption for cooperative and noncooperative MAC protocols are given in Fig. 6 (at low traffic load) and Fig. 7 (at high traffic load).Thanks to the channel diversity of the cooperative relay system, the source $S$ can still communicate with the destination $\mathrm{D}$ for a distance $d_{s d}=30 \mathrm{~m}$. This due to the fact with the help of the transmission from $\mathrm{R} 1$, the whole cooperative relay system can get the desired $B E R=10^{-5}$ for $d_{s d}=30 \mathrm{~m}$. On the one hand, the energy gain of cooperative relay over SISO decreases when using real MAC protocols, i.e. $33 \%$ (Fig. 6) instead of $35 \%$ (Fig. 5). As the traffic load increases (e.g. $\lambda=0.5$ ) this gain is only $22 \%$ (Fig. 7). The complex MAC protocol of the cooperative relay system with many packet transmissions for establishing the rendez-vous between nodes, obviously gets into trouble for high traffic load. So, unlike the consideration on energy consumption with an ideal MAC protocol in which cooperative relay system always gets energy gain over SISO system in long range transmission distance, our results show that the energy gain is significantly affected by MAC protocols and traffic load. However, the precise evaluation of energy consumption on a real platform (i.e. PowWow) still confirms the interest of cooperative relay system over SISO and multi-hop SISO 
system. On the other hand, in terms of latency, RIC-MAC protocol makes cooperative relay systems have low latency in comparison with multi-hop SISO sytems.

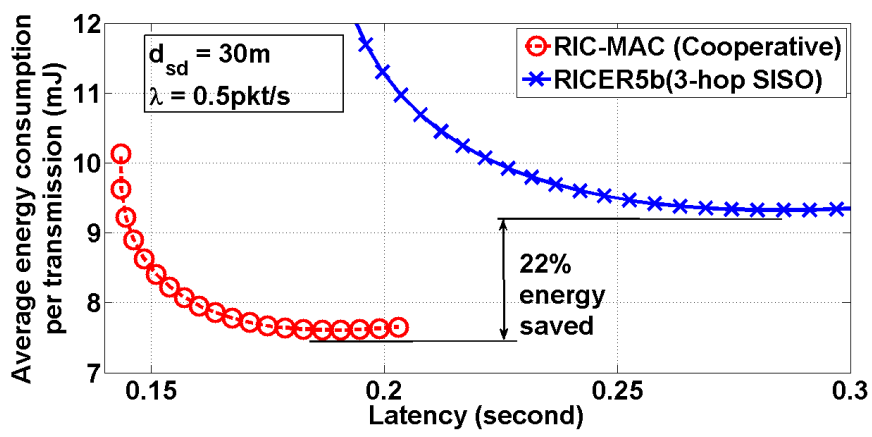

Fig. 7. Energy consumption with real MAC protocol (PowWow platform) in high traffic load networks

\section{Impact of traffic load on latency and energy}

The impact of traffic load on energy consumption and latency of RIC-MAC is shown in Fig. 8. With a fixed wakeup period $T$ (second), when the traffic load increases, the energy consumption increases as well. However, the latency is almost the same which means that RIC-MAC is robust to traffic load variations in terms of latency. Unless otherwise state, the following simulations are always considered as $d_{s d}=30 \mathrm{~m}$.

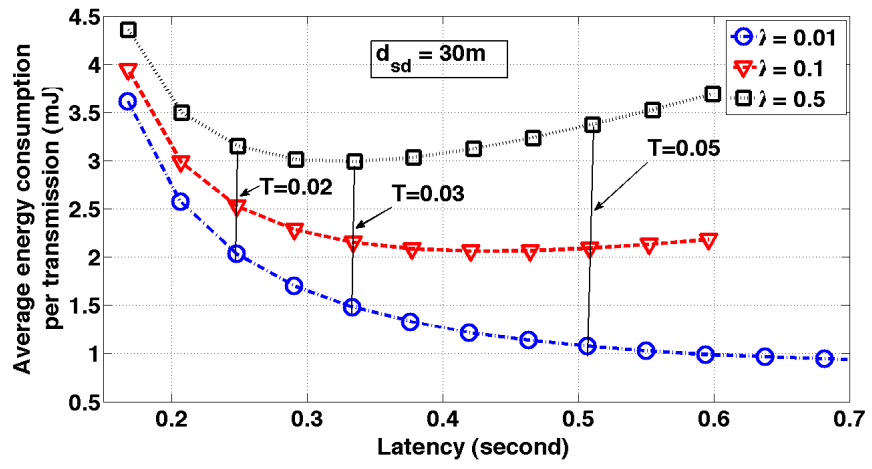

Fig. 8. Energy and latency of RIC-MAC as a function of $\lambda$.

It is well-known in the literature that it exists an optimal wakeup period $T^{o p t}$, which gives the best trade-off between the energy consumption and the latency of the communications, as mentioned in [21]. The comparison of energy consumption between cooperative RIC-MAC and 3hop RICER5b communications in the above results is taken at $T^{o p t}$. A thorough consideration of the energy consumption of the communications as a function of $\lambda$ at $T^{o p t}$ is given in Fig. 9. The energy consumption will increase quickly when $\lambda$ increases. As seen in the figure, cooperative RIC-MAC communications always have better performance than 3-hop RICER5b communications in terms of energy consumption no matter what $\lambda$ is.

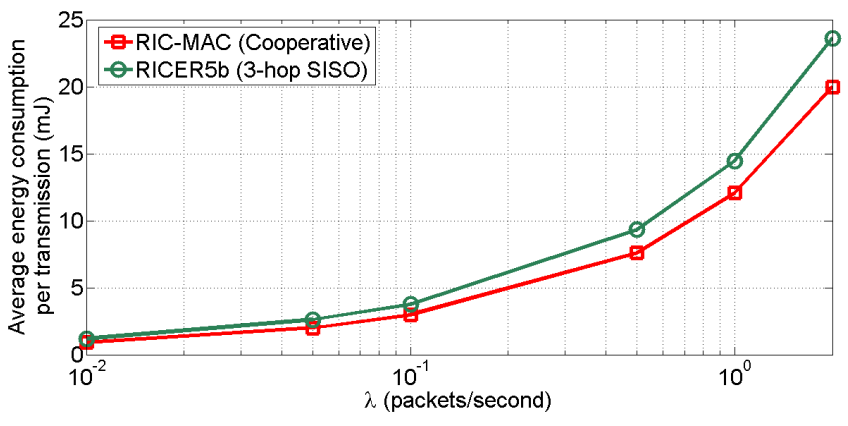

Fig. 9. Energy consumption of cooperative RIC-MAC and 3-hop RICER5b at $T^{\text {opt }}$ versus $\lambda$

On the other hand, Fig. 10 shows us another view on the energy consumption of the communications versus $\lambda$ in terms of the energy gain of cooperative RIC-MAC over 3-hop RICER5b. Seeing the increase of $\lambda$, the difference of energy consumption between the communications increases (Fig. 9) while the energy gain of cooperative RIC-MAC over 3-hop RICER5b decreases (Fig. 10). That means the lower $\lambda$ is, the more energy is saved by cooperative RIC-MAC compared to 3-hop RICER5b. Cooperative RIC-MAC is, therefore, more advantageous at low traffic modes.

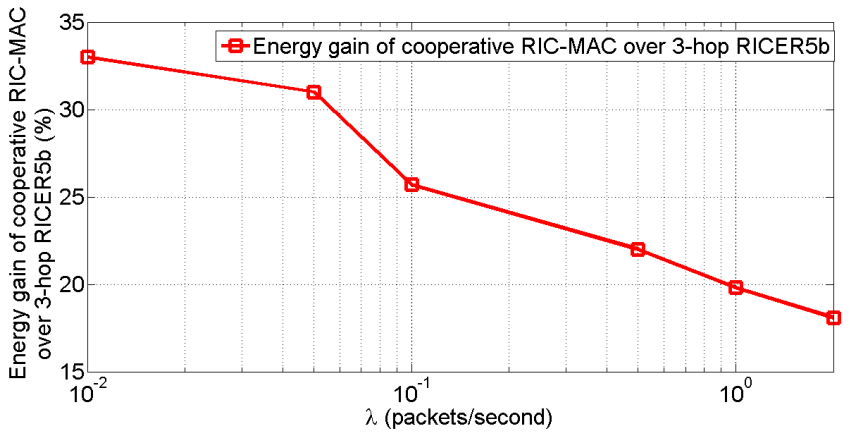

Fig. 10. Energy gain of cooperative RIC-MAC over 3-hop RICER5b at $T^{o p t}$ versus $\lambda$

Besides, the impact of traffic load on latency of the communications at $T^{o p t}$ is also considered in Fig. 11. When $\lambda$ increases, the latency of the communications logically decreases. As shown in Fig. 11, cooperative RIC-MAC communications provide better latency than 3-hop RICER5b at any value of $\lambda$.

\section{CONClusion}

In this paper, a thorough evaluation of the energy consumption of physical and MAC layers and of their cross influence is presented. However, the main objective of this paper is not to show the benefits of cooperative relay systems over SISO or multi-hop SISO systems which are very well-known in the literature. In contrast, the most important contribution of this study is to propose the RIC-MAC protocol, a new lowpower cooperative MAC protocol based on a receiver-initiated scheme. Besides, the impact of energy models (i.e. artificial 


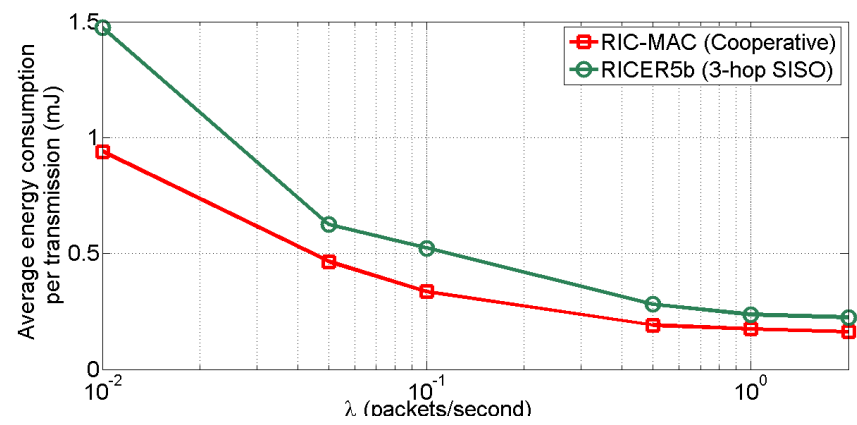

Fig. 11. Latency of cooperative RIC-MAC and 3-hop RICER5b at $T^{o p t}$ versus $\lambda$

model or real platform) and MAC protocols (i.e. ideal or real) on the energy consumption of the communications is also considered. Considering energy models extracted from a real WSN platform, the cooperative relay system still translates into energy gains as compared to multi-hop SISO systems at any network traffic load. However, the energy gain will be reduced when the network traffic load increases. Furthermore, in terms of latency, using RIC-MAC protocol in cooperative communications help to bring latency gain in comparison with multi-hop SISO communications. RIC-MAC protocol is finally shown to be robust to traffic load at fixed wakeup period.

\section{REFERENCES}

[1] J. Polastre, J. Hill, and D. Culler, "Versatile low power media access for wireless sensor networks," in Proceedings of the 2nd international conference on Embedded networked sensor systems. New York, NY, USA: ACM, 2004, pp. 95-107.

[2] M. Buettner, G. V. Yee, E. Anderson, and R. Han, "X-mac: a short preamble mac protocol for duty-cycled wireless sensor networks," in Proceedings of the 4th international conference on Embedded networked sensor systems. New York, NY, USA: ACM, 2006, pp. 307-320.

[3] A. El-Hoiydi and J.-D. Decotignie, "Wisemac: an ultra low power mac protocol for the downlink of infrastructure wireless sensor networks," in Ninth International Symposium on Computers and Communications, vol. 1, jun 2004, pp. $244-251$ Vol.1.

[4] E.-Y. Lin, J. Rabaey, and A. Wolisz, "Power-efficient rendez-vous schemes for dense wireless sensor networks," in IEEE International Conference on Communications, vol. 7, june 2004, pp. 3769 - 3776.

[5] E.-Y. Lin, J. Rabaey, S. Wiethoelter, and A. Wolisz, "Receiver initiated rendezvous schemes for sensor networks," in IEEE Global Telecommunications Conference, vol. 5, dec. 2005, pp. $3117-3122$.

[6] J. N. Laneman, G. W. Wornell, and D. N. C. Tse, "Cooperative diversity in wireless networks: Efficient protocols and outage behavior," IEEE Transactions on Information Theory, vol. 50, no. 12, pp. 3062-3080, Dec. 2004.
[7] T.-D. Nguyen, O. Berder, and O. Sentieys, "Cooperative miso and relay comparison in energy constrained wsns," in 71st Vehicular Technology Conference (VTC-Spring), 2010, pp. 1-5.

[8] T. Ahsin and S. Ben Slimane, "Energy efficiency using cooperative relaying," in IEEE 22nd International Symposium on Personal Indoor and Mobile Radio Communications (PIMRC), sept. 2011, pp. 1698 1702.

[9] G. de Oliveira Brante, M. Kakitani, and R. Souza, "Energy efficiency analysis of some cooperative and non-cooperative transmission schemes in wireless sensor networks," IEEE Transactions on Communications, vol. 59, no. 10, pp. $2671-2677$, october 2011.

[10] N. Guan, Y. Zhou, H. Liu, L. Tian, and J. Shi, "An energy efficient cooperative multicast transmission scheme with power control," in IEEE Global Telecommunications Conference, dec. 2011, pp. 1 -5.

[11] T.-D. Nguyen, O. Berder, and O. Sentieys, "Energy-efficient cooperative techniques for infrastructure-to-vehicle communications," IEEE Transactions on Intelligent Transportation Systems, vol. 12, no. 3, pp. 659 -668 , sept. 2011.

[12] J. Feng, R. Zhang, S. Ng, and L. Hanzo, "Relay selection for energyefficient cooperative media access control," in IEEE Wireless Communications and Networking Conference (WCNC), march 2011, pp. 287 $-292$.

[13] I. Stanojev, O. Simeone, Y. Bar-Ness, and D. H. Kim, "Energy efficiency of non-collaborative and collaborative hybrid-arq protocols," IEEE Transactions on Wireless Communications, vol. 8, no. 1, pp. 326 -335 , jan. 2009

[14] Y. Qi, R. Hoshyar, M. Imran, and R. Tafazolli, "The energy efficiency analysis of harq in hybrid relaying systems," in IEEE 73rd Vehicular Technology Conference (VTC Spring), may 2011, pp. 1 -5.

[15] P. Liu, Z. Tao, S. Narayanan, T. Korakis, and S. S. Panwar, "Coopmac: A cooperative mac for wireless lans," IEEE Journal on Selected Areas in Communications, vol. 25, no. 2, pp. 340 -354, february 2007.

[16] A. Crismani, F. Babich, and L. Hanzo, "Cross-layer solutions for cooperative medium access control protocols," in IEEE 71st Vehicular Technology Conference (VTC Spring), may 2010, pp. 1 -5.

[17] A. Ben Nacef, S.-M. Senouci, Y. Ghamri-Doudane, and A.-L. Beylot, "A cooperative low power mac protocol for wireless sensor networks," in IEEE International Conference on Communications (ICC), june 2011, pp. $1-6$.

[18] O. Berder and O. Sentieys, "Powwow : Power optimized hardware/software framework for wireless motes," in Proceedings of 23rd International Conference on Architecture of Computing Systems (ARCS), 2010, pp. $1-5$.

[19] S. Cui, A. Goldsmith, and A. Bahai, "Energy-efficiency of mimo and cooperative mimo techniques in sensor networks," IEEE Journal on Selected Areas in Communications, vol. 22, no. 6, pp. 1089 - 1098, aug. 2004.

[20] M. Otis, I. Chee, R. Lu, N. Pletcher, S. Gambini, and J. Rabaey, Lowpower electronics design. CRC Press, dec. 2003, ch. 31.

[21] O. Sentieys, O. Berder, P. Quemerais, and M. Cartron, "Wake-up interval optimization fo sensor networks with rendez-vous schemes," in Workshop on Design and Architectures for Signal and Image Processing (DASIP'07), November 2007. 